\title{
PTB/nPTB switch: a post-transcriptional mechanism for programming neuronal differentiation
}

\author{
Gabriela C. Coutinho-Mansfield, ${ }^{1}$ Yuanchao Xue, ${ }^{2}$ Yi Zhang, ${ }^{2}$ and Xiang-Dong Fu ${ }^{1,2,3}$ \\ ${ }^{1}$ Department of Cellular and Molecular Medicine, University of California at San Diego, La Jolla, California 92093, USA; \\ ${ }^{2}$ College of Life Sciences, Wuhan University, Wuhan 430072, People's Republic of China
}

Neuronal differentiation involves extensive reprogramming of gene expression. Many neuronal-specific genes are actively repressed in nonneuronal cells, while many others are induced in response to cell differentiation cues. Together these constitute the transcriptome in neurons to instruct specific neuronal functions (Rosenfeld et al. 2006). The transcriptome in neurons is further diversified by alternative splicing, arising from the expression of a number of neuronal-specific RNA-binding splicing regulators (Black and Grabowski 2003). In this issue of Genes \& Development, Boutz et al. (2007b) report a novel switch in the expression of a pair of related splicing regulators that occurs during neuronal differentiation. These proteins, known as polypyrimidine tractbinding proteins (PTB) and neural PTB (nPTB), are structurally and functionally similar, but PTB is widely expressed in nonneuronal cells, and $\mathrm{nPTB}$ is restricted to neurons. Remarkably, $25 \%$ of neuronally induced alternative splicing events detected by mRNA isoform-sensitive splicing arrays are estimated to result from the down-regulation of PTB, and the up-regulation of its neuronal-specific cousin $\mathrm{nPTB}$.

\section{Splicing control by PTB}

PTB has been extensively characterized for its sequencespecific binding to CU-rich motifs (Oberstrass et al. 2005), which are frequently part of the $3^{\prime}$ splice site in most constitutively spliced genes. Consequently, PTB binding often antagonizes the function of the essential splicing factor U2AF in the recognition of the 3' splice site (Singh et al. 1995). PTB-binding sites are also present in many discrete intronic locations to function as cisacting splicing silencer elements, and PTB has been identified as a negative splicing regulator of many alternative splicing events (Lin and Patton 1995; Chan and Black 1997; Wagner and Garcia-Blanco 2001). Recent biochemical analyses further revealed that PTB could alter

${ }^{3}$ Corresponding author.

E-MAIL xdfu@ucsd.edu; FAX (858) 822-6920.

Article is online at http://www.genesdev.org/cgi/doi/10.1101/gad.1575607. the recognition of splicing signals that are far away from its binding sites, indicating an extended competition between the splicing machinery and the PTB-induced splicing silencing complexes (Izquierdo et al. 2005; Sharma et al. 2005; Spellman and Smith 2006).

While the role of PTB in regulated splicing has been well documented on specific minigene models, the breadth of its involvement in regulated splicing has remained undetermined until now. Using RNA interference (RNAi) in combination with splicing arrays, Boutz et al. (2007b) found that PTB knockdown in N2A neuroblastoma cells indeed altered the splicing pattern of many genes, identifying PTB as a key splicing regulator in mammalian cells. A large percentage $(83 \%)$ of the splicing changes triggered by PTB RNAi were also detected in differentiating P19 cells upon neuronal induction by retinoic acid, consistent with the strong suppression of PTB expression during the induction. Strikingly, judging from the common changes from PTB RNAi in N2A cells, altered splicing in response to PTB inactivation could account for $\sim 25 \%$ of the induced alternative splicing in differentiated P19 cells. This suggests that PTB down-regulation is a major factor in establishing the splicing-mediated neuronal differentiation program. Mechanistically, many of the neuronally induced switches in isoform expression appear to be directly mediated by PTB in nonneuronal cells, because of the presence of the expected CU-rich motifs near the alternative exons. Other splicing events may be indirectly induced by PTBregulated splicing of other splicing regulators. Another recent splicing array study revealed that systems where one alternative splicing factor is controlled by another splicing regulator appear to be common, and may create a variety of feedback and feed forward regulation at the splicing level (Ni et al. 2007).

\section{PTB regulation of $\mathrm{nPTB}$ expression}

One of the major functional consequences of PTB knockdown is actually the induction of a highly related gene transcript encoding $\mathrm{nPTB}$, which has been previously 
shown to be a brain-specific counterpart of PTB (Ashiya and Grabowski 1997; Kikuchi et al. 2000; Markovtsov et al. 2000; Polydorides et al. 2000). In the current study, detailed immunocytochemistry further showed in brain sections that PTB is mainly expressed in cells of nonneuronal lineages, while $\mathrm{nPTB}$ protein is largely restricted to neurons. Interestingly, $\mathrm{nPTB}$ mRNA was readily detectable in a variety of nonneuronal cell types and down-regulation of PTB induced nPTB protein synthesis by at least two post-transcriptional mechanisms.

It is known that PTB feedback regulates its own expression by binding to intronic silencers around the alternative PTB exon 11 to promote its skipping (Wollerton et al. 2004). As illustrated in Figure 1, PTB binding causes skipping of exon 11, resulting in a reading frame shift and the premature termination of translation. This premature termination induces degradation of the aberrantly spliced PTB transcript by the nonsense-mediated RNA decay (NMD) pathway (Maquat 2005). This mechanism is thought to maintain homeostasis of PTB expression in nonneuronal cells (Wollerton et al. 2004). PTB is now found to operate similarly on the nPTB pre-mRNA through ultraconserved cis-acting elements in the introns surrounding the nPTB alternative exon 10, which is equivalent to exon 11 in PTB. In response to PTB

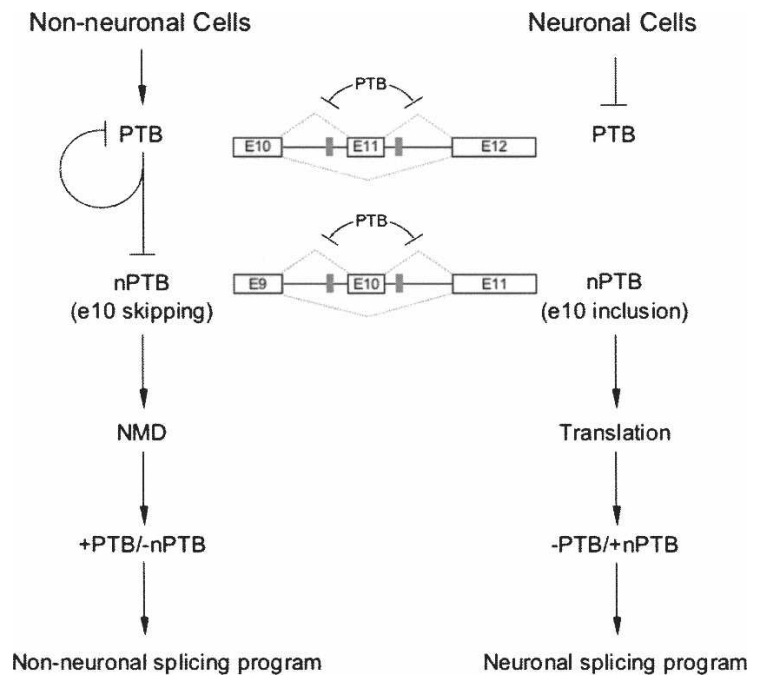

Figure 1. A neuronal-specific splicing program induced by the $\mathrm{PTB} / \mathrm{nPTB}$ switch. $P T B$ is transcriptionally active in nonneuronal cells and is silent in neuronal cells. In contrast, $n P T B$ is actively transcribed in both nonneuronal and neuronal cells. PTB represses the inclusion of its own alternative exon 11 by binding to the intronic splicing silencers (gray bar) on both sides of exon 11, a mechanism thought to maintain PTB homeostasis in nonneuronal cells. PTB was found to act in part on $\mathrm{nPTB}$ through the intronic splicing silencers (gray bar) adjacent to alternative exon 10 (which is homologous to exon 11 in PTB). As a result, exon 10 is skipped in nonneuronal cells, introducing a premature stop codon that in turn triggers NMD of the transcript. This post-transcriptional regulation is coupled with an unknown translational inhibition mechanism to effectively block nPTB expression in nonneuronal cells. In neurons, the absence of PTB allows nPTB expression. down-regulation by RNAi in N2A or other cells, nPTB splicing was derepressed to include more exon 10. It appears that this same mechanism will affect nPTB after PTB is lost during retinoic acid induction in P19 cells. Furthermore, inhibition of NMD action by the translational inhibitor Cycloheximide or impairment of the NMD machinery by RNAi against Upf1 led to the stabilization of the exon 10 skipped $\mathrm{nPTB}$ in a variety of cell types. This demonstrates that the coupling of alternative splicing to NMD is suppressing nPTB expression in nonneuronal cells. Since PTB is controlling both PTB and $\mathrm{nPTB}$ alternative splicing, it will be interesting to determine whether $\mathrm{nPTB}$ has a similar activity that contributes to the repression of PTB expression, and perhaps the maintenance of $\mathrm{nPTB}$ expression in post-mitotic neurons.

Apparently, the negative regulation of $\mathrm{nPTB}$ by PTB at the splicing level is only part of the story, as the level of nPTB protein was often more dramatically increased after PTB knockdown than the amount of the exon 10containing $\mathrm{nPTB}$ mRNA. This indicates that the expression of nPTB may also be regulated at the level of protein synthesis, and that PTB may play a direct or indirect role in the regulation. While the mechanism remains elusive at this point, PTB has been implicated in the regulation of translation (Irwin et al. 1997; Hunt and Jackson 1999), and thus, it is possible that PTB may act directly in repressing $\mathrm{nPTB}$ translation. Alternatively, PTB may affect the expression of other translational regulators to indirectly modulate nPTB protein synthesis. Consistent with the possibility that these splicing regulators may be regulated in the cytoplasm, recent studies indicate that both PTB and $\mathrm{nPTB}$ are subject to translational regulation by microRNAs in muscles and neurons (Lim et al. 2005; Boutz et al. 2007a). It will therefore be interesting in its own right to investigate how $\mathrm{PTB}$ and $\mathrm{nPTB}$ may control the translation of their own mRNAs in a feedback regulatory loop and the potential involvement of microRNAs in the process.

\section{Splicing reprogramming induced by the PTB/nPTB switch}

$\mathrm{nPTB}$ is structurally related to $\mathrm{PTB}$, indicating that they may have overlapping, but distinct target specificity in regulated splicing. The related function of $\mathrm{PTB}$ and $\mathrm{nPTB}$ in splicing regulation has been characterized on several minigene models (Ashiya and Grabowski 1997; Markovtsov et al. 2000). Using the splicing array coupled with single and double RNAi knockdown, the current report demonstrates that the two protein paralogs indeed exhibit partially redundant functions in the regulation of alternative splicing. It is, however, important to emphasize their differences: The alternative exons regulated by PTB differ significantly from those regulated by nPTB, and double knockdown gives rise to a further distinct splicing profile. Together, these results clearly illustrate the different target spectra for $\mathrm{PTB}$ and $\mathrm{nPTB}$, and it is the down-regulation of PTB and induction of $\mathrm{nPTB}$ that commits cells to a unique and neuronal-specific splicing program. 
The redundant and distinct functions associated with PTB and $\mathrm{nPTB}$ have important biological implications for the splicing program in both neuronal and nonneuronal cells. Alternative splicing regulated by $\mathrm{PTB} / \mathrm{nPTB}$ may be critical for a variety of cellular functions. Thus, to prevent a complete loss of PTB function, mammalian cells have evolved the mechanism to maintain a level of $\mathrm{nPTB}$ transcription to permit a rapid response to potential malfunction of PTB. In developing neurons, on the other hand, the PTB/nPTB switch appears to represent a key post-transcriptional reprogramming event. Instead of dramatically altering the transcriptional program, the $\mathrm{PTB} / \mathrm{nPTB}$ switch may fine tune the existing program to meet the functional requirements of the nervous system. Further studies on single- and double-knockout animal models will address these important biological questions (see below).

It is interesting to note that, in addition to the PTB/ nPTB switch from nonneuronal to neuronal cells, the splicing program in different neuronal cell types may be further fine tuned by the differential expression of other pairs of neuronal-specific splicing regulators. For example, both Nova-1 and Nova-2 are neuronal splicing factors, whose expression in postnatal brain seems largely reciprocal, most obviously in the neocortex where Nova-2 is nearly exclusively expressed (Yang et al. 1998). Furthermore, because Nova-1 is also autoregulated at the splicing level (Dredge et al. 2005), Nova-1 and Nova-2 may undergo both feedback and feed-forward regulation similar to $\mathrm{PTB}$ and $\mathrm{nPTB}$. The resulting Noval/2 switch in different brain regions may relay the $\mathrm{PTB} / \mathrm{nPTB}$ switch from nonneuronal to neuronal cells to reprogram alternative splicing in different subtypes of neurons.

\section{Transcriptional and post-transcriptional induction} of the neuronal splicing program

One of the most striking findings from the current work is that the $\mathrm{PTB} / \mathrm{nPTB}$ switch affects such a large fraction of neuronally induced alternative splicing events. Splicing arrays have also been used to characterize splicing defects in Nova knockout brains (Ule et al. 2005a), although it is unclear how many Nova-dependent splicing events are induced in the P19 system. It may be that a large fraction of neuronal-specific splicing events are controlled by a panel of neuronal-specific splicing regulators that have already been biochemcially and/or genetically characterized, such as Nova (Ule et al. 2005a,b, 2006), Fox (Jin et al. 2003; Auweter et al. 2006), and Hu (Perrone-Bizzozero and Bolognani 2002; Zhu et al. 2006) families of proteins. These neuronal-specific splicing regulators, as well as more general splicing regulators, are likely to act in a combinatorial fashion with large overlapping target sets to establish the alternative splicing program in neurons.

Important questions that remain as to how PTB expression is actively suppressed, and how of the other neuronal-specific splicing regulators are induced in neurons, will hold the key to our understanding of the tran- scriptome in neurons. Previous studies have established that a large number of neuronal genes are specifically repressed at the transcriptional level in nonneuronal cells by the REST/CoREST complex (Andres et al. 1999; Ballas et al. 2001, 2005; Lunyak et al. 2002). It will be interesting to determine whether any neuronal-specific splicing regulators are coordinately regulated by the RSET/CoREST complex. MicroRNAs may also play a big part in this regulation (Johnston et al. 2005; Boutz et al. 2007a). The PTB/nPTB switch certainly adds an interesting post-transcriptional mechanism for the induction and maintenance of neuronal-specific genes.

\section{The road ahead: linking regulated splicing to neuronal phenotype}

One challenge for future research will be to determine whether the PTB/nPTB switch is essential for neuronal differentiation, or is one of the events that accompany neuronal differentiation. Judging from their widespread effects on the splicing program,, the switch may play a key role in neuronal differentiation itself. This question may be addressed by expressing exogenous PTB from a constitutive promoter in P19 cells to determine whether forced PTB expression might be sufficient to prevent P19 cell differentiation into neurons. Alternatively, the PTB/ nPTB switch may modulate specific neuronal functions, but the switch itself may not be essential to commit cells to the neuronal differential pathway. If this is the case, it will be interesting to determine whether $\mathrm{APTB}$ is still induced in presence of exogenous PTB during neuronal differentiation. This experiment will confirm whether $\mathrm{nPTB}$ induction is solely regulated by $\mathrm{PTB}$ down-regulation.

Splicing arrays have revealed numerous neuronal-specific mRNA isoforms regulated by Nova (Ule et al. 2005a), and now by PTB and nPTB. Many of those detected isoforms appear directly relevant to neuronal functions as discussed by Ule et al. (2005a) and Boutz et al. (2005b). The link between regulated splicing and neuronal phenotypes can be established by developing specific functional assays to correlate a specific expressed isoform to a defined functional consequence in neurons. Technically, this is challenging, but it has been done in other experimental systems where a specific splicing regulator is either overexpressed or genetically inactivated and a specific alternative splicing event is directly linked to a measurable phenotype (e.g., see Ghigna et al. 2005; Xu et al. 2005; Karni et al. 2007). Antisense technology and isoform-specific inactivation by RNAi also provide powerful tools for functional dissection of alternative splicing induced by the PTB/nPTB switch (GarciaBlanco et al. 2004; Hua et al. 2007). Future genotype/ phenotype studies of regulated splicing during development will clearly benefit from the construction of genetically engineered cell and animal models.

Besides regulated splicing, it is important to keep in mind that PTB is also known to play roles in other aspects of RNA metabolism, including 3' end formation (Castelo-Branco et al. 2004; Le Sommer et al. 2005), and 
the regulation of RNA stability and translation (Irwin et al. 1997; Hunt and Jackson 1999; Knoch et al. 2004). By extrapolation, nPTB may play both similar and distinct roles in these processes, which may also contribute to important neuronal functions. The current work in conjunction with the published work on Nova and general splicing factors, such as SR proteins, illustrates a general experimental approach to understand regulated splicing in development and disease (Xu and Fu 2005; Möröy and Heyd 2007).

\section{References}

Andres, M.E., Burger, C., Peral-Rubio, M.J., Battaglioli, E., Anderson, M.E., Grimes, J., Dallman, J., Ballas, N., and Mandel, G. 1999. CoREST: A functional corepressor required for regulation of neural-specific gene expression. Proc. Natl. Acad. Sci. 96: 9873-9878.

Ashiya, M. and Grabowski, P.J. 1997. A neuron-specific splicing switch mediated by an array of pre-mRNA repressor sites: Evidence of a regulatory role for the polypyrimidine tract binding protein and a brain-specific PTB counterpart. RNA 3: 996-1015.

Auweter, S.D., Fasan, R., Reymond, L., Underwood, J., Black, D.L., Pitsch, S., and Allain, F. 2006. Molecular basis of RNA recognition by the human alternative splicing factor Fox-1. EMBO I. 25: 163-173.

Ballas, N., Battaglioli, E., Atouf, F., Andres, M.E., Chenoweth, J., Anderson, M.E., Burger, C., Moniwa, M., Davie, J.R., Bowers, W.J., et al. 2001. Regulation of neuronal traits by a novel transcriptional complex. Neuron 31: 353-365.

Ballas, N., Grunseich, C., Lu, D.D., Speh, J.C., and Mandel, G. 2005. REST and its corepressors mediate plasticity of neuronal gene chromatin throughout neurogenesis. Cell 121: 645-657.

Black, D.L. and Grabowski, P.J. 2003. Alternative pre-mRNA splicing and neuronal function. Prog. Mol. Subcell. Biol. 31: $187-216$

Boutz, P.L., Chawla, G., Stoilov, P., and Black, D.L. 2007a. MicroRNAs regulate the expression of the alternative splicing factor nPTB during muscle development. Genes \& Dev. 21: 71-84.

Boutz, P.L., Stoilov, P., Li, Q., Lin, C.-H., Chawla, G., Ostrow, K., Shiue, L., Ares Jr., M., and Black, D.L. 2007b. A posttranscriptional regulatory switch in polypyrimidine tractbinding proteins reprograms alternative splicing in developing neurons. Genes \& Dev. (this issue), doi: 10.1101/ gad.1558107.

Castelo-Branco, P., Furger, A., Wollerton, M., Smith, C., Moreira, A., and Proudfoot, N. 2004. Polypyrimidine tract binding protein modulates efficiency of polyadenylation. Mol. Cell. Biol. 24: 4174-4183.

Chan, R.C. and Black, D.L. 1997. The polypyrimidine tract binding protein binds upstream of neural cell-specific c-src exon N1 to repress the splicing of the intron downstream. Mol. Cell. Biol. 17: 4667-4676.

Dredge, B.K., Stefani, G., Engelhard, C.C., and Darnell, R.B. 2005. Nova autoregualtion reveals dual functions in neuronal splicing. $E M B O$ J. 24: 1608-1620.

Garcia-Blanco, M.A., Baraniak, A.P., and Lasda, E.L. 2004. Alternative splicing in disease and therapy. Nat. Biotechnol. 22: 535-546.

Ghigna, C., Giordano, S., Shen, H., Benvenuto, F., Castiglioni, F., Comoglio, P.M., Green, M.R., Riva, S., and Biamonti, G.
2005. Cell motility is controlled by SF2/ASF through alternative splicing of the Ron protooncogene. Mol. Cell 20: 881890.

Hua, Y., Vickers, T.A., Baker, B.F., Bennett, C.F., and Krainer, A.R. 2007. Enhancement of SMN2 exon 7 inclusion by antisense oligonucleotides targeting the exon. PLOS Biol. 5: e73. doi: 10.1371/journal.pbio.0050073.

Hunt, S.L. and Jackson, R.J. 1999. Polypyrimidine-tract binding protein $(\mathrm{PTB})$ is necessary, but not sufficient, for efficient internal initiation of translation of human rhinovirus-2 RNA. RNA 5: 344-359.

Irwin, N., Baekelandt, V., Goritchenko, L., and Benowitz, L.I. 1997. Identification of two proteins that bind to a pyrimidine-rich sequence in the 3 '-untranslated region of GAP-43 mRNA. Nucleic Acids Res. 25: 1281-1288.

Izquierdo, J.M., Majos, N., Bonnal, S., Martinez, C., Castelo, R., Guigo, R., Bilbao, D., and Valcarcel, J. 2005. Regulation of Fas alternative splicing by antagonistic effects of TIA-1 and PTB on exon definition. Mol. Cell 19: 475-484.

Jin, Y., Suzuki, H., Maegawa, S., Endo, H., Sugano, S., Hashimoto, K., Yasuda, K., and Inoue, K. 2003. A vertebrate RNAbinding protein Fox-1 regulates tissue-specific splicing via the pentanucleotide GCAUG. EMBO J. 22: 905-912.

Johnston Jr., R.J., Chang, S., Etchberger, J.F., Ortiz, C.O., and Hobert, O. 2005. MicroRNAs acting in a double-negative feedback loop to control a neuronal cell fate decision. Proc. Natl. Acad. Sci. 102: 12449-12454.

Karni, R., de Stanchina, E., Lowe, S.W., Sinha, R., Mu, D., and Krainer, A.R. 2007. The gene encoding the splicing factor SF2/ASF is a proto-oncogene. Nat. Struct. Mol. Biol. 14: 185193.

Kikuchi, T., Ichikawa, M., Arai, J., Tateiwa, H., Fu, L., Higuchi, K., and Yoshimura, N. 2000. Molecular cloning and characterization of a new neuron-specific homologue of rat polypyrimidine tract binding protein. J. Biochem. 128: 811-821.

Knoch, K., Bergert, H., Borgonovo, B., Saeger, H., Altkrüger, A., Verkade, P., and Solimena, M. 2004. Polypyrimidine tractbinding protein promotes insulin secretory granule biogenesis. Nat. Cell Biol. 6: 207-214.

Le Sommer, C., Lesimple, M., Mereau, A., Menoret, S., Allo, M.R., and Hardy, S. 2005. PTB regulates the processing of a 3 '-terminal exon by repressing both splicing and polyadenylation. Mol. Cell. Biol. 25: 9595-9607.

Lim, L.P., Lau, N.C., Garrett-Engele, P., Grimson, A., Schelter, J.M., Castle, J., Bartel, D.P., Linsley, P.S., and Johnson, J.M. 2005. Microarray analysis shows that some microRNAs downregulate large numbers of target mRNAs. Nature 433: 769-773.

Lin, C.-H. and Patton, J.G. 1995. Regulation of alternative 3' splice site selection by constitutive splicing factors. RNA 1: 234-245.

Lunyak, V.V., Burgess, R., Prefontaine, G.G., Nelson, C., Sze, S.H., Chenoweth, J., Schwartz, P., Pevzner, P.A., Glass, C., Mandel, G., et al. 2002. Corepressor-dependent silencing of chromosomal regions encoding neuronal genes. Science $\mathbf{2 9 8}$ 1747-1752.

Maquat, L.E. 2005. Nonsense-mediated mRNA decay in mammals. J. Cell Sci. 118: 1773-1776.

Markovtsov, V., Nikolic, J.M., Goldman, J.A., Turck, C.W., Chou, M.Y., and Black, D.L. 2000. Cooperative assembly of an hnRNP complex induced by a tissue-specific homolog of polypyrimidine track binding protein. Mol. Cell. Biol. 20: 7463-7479.

Möröy, T. and Heyd, F. 2007. The impact of alternative splicing in vivo: Mouse models show the way. RNA doi: 10.1261/ rna. 554607 . 
Ni, J.Z., Grate, L., Donohue, J.P., Preston, C., Nobida, N., O'Brien, G., Shiue, L., Clark, T.A., Blume, J.E., and Ares Jr., M. 2007. Ultraconserved elements are associated with homeostatic control of splicing regulators by alternative splicing and nonsense-mediated decay. Genes \& Dev. 21: 708718.

Oberstrass, F.C., Auweter, S.D., Erat, M., Hargous, Y., Henning, A., Wenter, P., Reymond, L., Amir-Ahmady, B., Pitsch, S., Black, D.L., et al. 2005. Structure of PTB bound to RNA: Specific binding and implications for splicing regulation. Science 309: 2054-2057.

Perrone-Bizzozero, N. and Bolognani, F. 2002. Role of HuD and other RNA-binding proteins in neural development and plasticity. J. Neurosci. Res. 68: 121-126.

Polydorides, A.D., Okano, H.J., Yang, Y.Y., Stefani, G., and Darnell, R.B. 2000. A brain-enriched polypyrimidine tract-binding protein antagonizes the ability of Nova to regulate neuron-specific alternative splicing. Proc. Natl. Acad. Sci. 97: 6350-6355.

Rosenfeld, M.G., Lunyak, V.V., and Glass, C.K. 2006. Sensors and signals: A coactivator/corepressor/epigenetic code for integrating signal-dependent programs of transcriptional response. Genes \& Dev. 20: 1405-1428.

Sharma, S., Falick, A.M., and Black, D.L. 2005. Polypyrimidine tract binding protein blocks the $5^{\prime}$ splice site-dependent assembly of U2AF and the prespliceosomal E complex. Mol. Cell 19: 485-496.

Singh, R., Valcarcel, J., and Green, M.R. 1995. Distinct binding specificities and functions of higher eukaryotic polypyrimidine tract-binding proteins. Science 268: 1173-1176.

Spellman, R. and Smith, C.W. 2006. Novel modes of splicing repression by PTB. Trends Biochem. Sci. 31: 73-76.

Ule, J., Ule, A., Spencer, J., Williams, A., Hu, J.S., Cline, M., Wang, H., Clark, T., Fraser, C., Ruggiu, M., et al. 2005a. Nova regulates brain-specific splicing to shape the synapse. Nat. Genet. 37: 844-852.

Ule, J., Jensen, K., Mele, A., and Darnell, R.B. 2005b. CLIP: A method for identifying protein-RNA interaction sites in living cells. Methods 37: 376-386.

Ule, J., Stefani, G., Mele, A., Ruggiu, M., Wang, X., Taneri, B., Gaasterland, T., Blencowe, B.J., and Darnell, R.B. 2006. An RNA map predicting Nova-dependent splicing regulation. Nature 444: 580-586.

Wagner, E.J. and Garcia-Blanco, M.A. 2001. Polypyrimidine tract binding protein antagonizes exon definition. Mol. Cell. Biol. 21: 3281-3288.

Wollerton, M.C., Gooding, C., Wagner, E.J., Garcia-Blanco, M.A., and Smith, C.W. 2004. Autoregulation of polypyrimidine tract binding protein by alternative splicing leading to nonsense-mediated decay. Mol. Cell 13: 91-100.

$\mathrm{Xu}, \mathrm{X}$. and Fu, X.D. 2005. Conditional knockout mice to study alternative splicing in vivo. Methods 37: 387-392.

Xu, X., Yang, D., Ding, J.-H., Wang, W., Chu, P.-H., Dalton, N.D., Wang, H.-Y., Bermingham Jr., J.R., Ye, Z., Liu, F., et al. 2005. ASF/SF2-regulated postnatal reprogramming of CaMKII $\delta$ alternative splicing modulates excitation-contraction coupling in cardiac muscle. Cell 120: 59-72.

Yang, Y.Y., Yin, G.L., and Darnell, R.B. 1998. The neuronal RNA-binding protein Nova-2 is implicated as the autoantigen targeted in PMA patients with dementia. Proc. Natl. Acad. Sci. 95: 13254-13259.

Zhu, H., Hasman, R.A., Barron, V.A., Luo, G., and Lou, H. 2006. A nuclear function of $\mathrm{Hu}$ proteins as neuron-specific alternative RNA processing regulators. Mol. Biol. Cell 17: 51055114. 


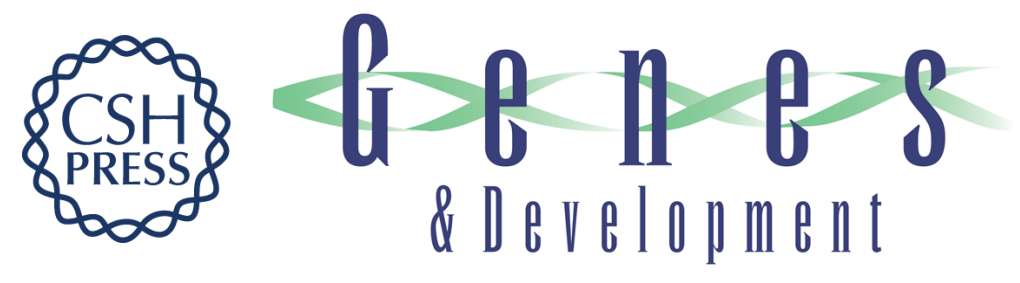

\section{PTB/nPTB switch: a post-transcriptional mechanism for programming neuronal differentiation}

Gabriela C. Coutinho-Mansfield, Yuanchao Xue, Yi Zhang, et al.

Genes Dev. 2007, 21:

Access the most recent version at doi:10.1101/gad.1575607
Related Content A post-transcriptional regulatory switch in polypyrimidine tract-binding proteins reprograms alternative splicing in developing neurons
Paul L. Boutz, Peter Stoilov, Qin Li, et al.
Genes Dev. July , 2007 21: 1636-1652
References This article cites 46 articles, 25 of which can be accessed free at:
http://genesdev.cshlp.org/content/21/13/1573.full.html\#ref-list-1
Articles cited in:
http://genesdev.cshlp.org/content/21/13/1573.full.html\#related-urls

\section{License}
Email Alerting
Service
Receive free email alerts when new articles cite this article - sign up in the box at the top right corner of the article or click here.

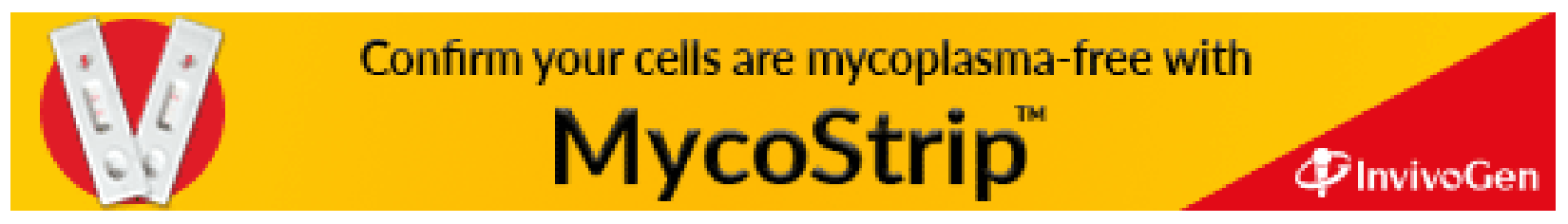

